Original article

\title{
A comparative test of ixodid tick identification by a network of European researchers
}

\author{
A. Estrada-Peñaa ${ }^{a, *}$, G. D’Amico ${ }^{b}$, A.M. Palomar ${ }^{c}$, M. Dupraz $^{\mathrm{d}}$, M. Fonville $^{\mathrm{e}}$, D. Heylen $^{\mathrm{f}}$, \\ Habela M.A. ${ }^{g}$, S. Hornok ${ }^{\mathrm{h}}$, L. Lempereur ${ }^{\mathrm{i}}$, M. Madder $^{\mathrm{j}}$, M.S. Núncio ${ }^{\mathrm{k}}$, D. Otranto ${ }^{\mathrm{l}}$, \\ M. Pfaffle ${ }^{\mathrm{m}}$, O. Plantard ${ }^{\mathrm{n}}$, M.M. Santos-Silva ${ }^{\mathrm{k}}$, H. Sprong $^{\mathrm{e}}$, Z. Vatansever $^{\mathrm{O}}$, L. Vial $^{\mathrm{p}}$, \\ A.D. Mihalca ${ }^{\text {b }}$
}

a Department of Animal Health, Faculty of Veterinary Medicine, Miguel Servet 177, 50013, Zaragoza, Spain

${ }^{\mathrm{b}}$ University of Agricultural Sciences and Veterinary Medicine Cluj-Napoca, Department of Parasitology and Parasitic Diseases, Cluj-Napoca, Romania

c Center of Rickettsiosis and Arthropod-Borne Diseases, Hospital San Pedro-CIBIR, Logroño, La Rioja, Spain

d MIVEGEC UMR 5290 IRD-CNRS-UM1-UM2 Centre IRD, 911 Avenue Agropolis, BP 64501, 34394 Montpellier, France

e Laboratory for Zoonoses and Environmental Microbiology, National Institute for Public Health and Environment (RIVM), Bilthoven, The Netherlands

${ }^{\mathrm{f}}$ University of Antwerp, Department of Biology, Evolutionary Ecology Group, Antwerpen, Belgium

g Parasitology \& Parasitic Diseases, Department of Animal Health, Faculty of Veterinary Medicine, University of Extremadura,10071, Cáceres, Spain

h University of Veterinary Medicine, Department of Parasitology and Zoology, Istvan u. 2., 1078 Budapest, Hungary

${ }^{i}$ Laboratory of Parasitology and Parasitic Diseases, Faculty of Veterinary Medicine, University of Liège, Liège, Belgium

${ }^{\mathrm{j}}$ Department of Veterinary Tropical Diseases, Faculty Veterinary Science, University of Pretoria, Private Bag X04, Onderstepoort, 0110, South Africa

${ }^{\mathrm{k}}$ Instituto Nacional de Saúde Dr. Ricardo Jorge, Centro de Estudos de Vectores e Doenças Infecciosas Dr. Francisco Cambournac, Av. da Liberdade, 5 ,

2965-575, Águas de Moura, Portugal

${ }^{1}$ Department of Veterinary Medicine, University of Bari, Str. prov. per Casamassima km 3, 70010 Valenzano, Bari, Italy

${ }^{\mathrm{m}}$ Karlsruhe Institute of Technology, Zoological Institute, Department of Ecology and Parasitology, 76131 Karlsruhe, Germany

n BIOEPAR, INRA, Oniris, La Chantrerie, 44307, Nantes, France

${ }^{\circ}$ Kafkas University, Faculty of Veterinary Medicine, Department of Parasitology, Kars, Turkey

P CIRAD, UMR CMAEE, F-34398 Montpellier, France

\section{A R T I C L E I N F O}

\section{Article history:}

Received 29 August 2016

Received in revised form 4 March 2017

Accepted 6 March 2017

Available online 8 March 2017

\section{Keywords:}

Comparative test

Identification

Morphology

Molecular

Ixodid ticks

Western Palearctic

\begin{abstract}
A B S T R A C T
This study reports the results of a comparative test of identification of ticks occurring in Western Europe and Northern Africa. A total of 14 laboratories were voluntarily enrolled in the test. Each participant received between 22 and 25 specimens of adult and nymphal ticks of 11 species: Dermacentor marginatus, D. reticulatus, Haemaphysalis punctata, Hyalomma lusitanicum, Hy. marginatum, Ixodes ricinus, I. hexagonus, Rhipicephalus annulatus, $R$. bursa, $R$. rossicus, and/or $R$. sanguineus s.l. Ticks were morphologically identified by three of the co-authors and the identification confirmed by a fourth co-author who used molecular methods based on several genes. Then ticks were randomly selected and blindly distributed among participants, together with a questionnaire. Only specimens collected while questing and, if possible, in the same survey, were circulated. Because of the random nature of the test, a participant could receive several specimens of the same species. Species in the different genera had variable misidentification rates (MR) of 7\% (Dermacentor), 14\% (Ixodes), 19\% (Haemaphysalis), 36\% (Hyalomma), and 54\% (Rhipicephalus). Within genera, the MR was also variable ranging from $5.4 \%$ for $I$. ricinus or $7.4 \%$ for $D$. marginatus or D. reticulatus to $100 \%$ for $R$. rossicus. The test provided a total misidentification rate of $29.6 \%$ of the species of ticks. There are no significant differences in MR according to the sex of the tick. Participants
\end{abstract}

\footnotetext{
* Corresponding author.

E-mail addresses: aestrada@unizar.es (A. Estrada-Peña), gianluca.damico@usamvcluj.ro (G. D’Amico), ampalomar@riojasalud.es (A.M. Palomar), marlene.dupraz@ird.fr (M. Dupraz), manoj.fonville@rivm.nl (M. Fonville), Dieter.Heylen@uantwerpen.be (D. Heylen), mahabela@unex.es (M.A. Habela),

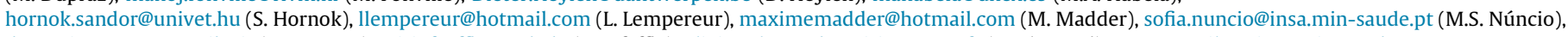
domenico.otranto@uniba.it (D. Otranto), miripfaeffle@web.de (M. Pfaffle), olivier.plantard@oniris-nantes.fr (O. Plantard), m.santos.silva@insa.min-saude.pt (M.M. Santos-Silva), hein.sprong@rivm.nl (H. Sprong), zativet@gmail.com (Z. Vatansever), laurence.vial@cirad.fr (L.Vial), amihalca@usamvcluj.ro (A.D. Mihalca).
} 
were requested to perform a second round of identifications on the same set of ticks, using only purposely prepared keys (without illustrations), circulated to the enrolled participants, including 2 species of the genus Dermacentor, 8 of Haemaphysalis, 10 of Hyalomma, 23 of Ixodes, and 6 of Rhipicephalus. The average MR in the second round was 28\%: 0\% (Dermacentor), 33\% (Haemaphysalis), 30\% (Hyalomma) 18\% (Ixodes), and 50\% (Rhipicephalus). Species which are not reported in the countries of a participating laboratory had always highest MR, i.e. purely Mediterranean species had highest MR by laboratories in Central and Northern Europe. Participants expressed their concerns about a correct identification for almost $50 \%$ of the ticks of the genera Hyalomma and Rhipicephalus. The results revealed less than total confidence in identifying the most prominent species of ticks in the Western Palearctic, and underpin the need for reference libraries for specialists involved in this task. Results also showed that a combination of certain genes may adequately identify the target species of ticks.

(c) 2017 Elsevier GmbH. All rights reserved.

\section{Background}

Ticks are known to transmit a large variety of pathogens of medical and veterinary concern and are among the most important disease-transmitting arthropods (Estrada-Peña and de la Fuente, 2014). Field studies on ticks should be based on a correct identification of the specimens collected, as a crucial step in a chain of microbiological or epidemiological studies. The identification has been commonly done only by morphological examination, and the use of "molecular only" protocols are still uncommon in Europe. For example, in a recent literature review on the occurrence of ticks and tick-borne pathogens in Europe (Maioli et al., 2012), only studies that used morphological keys for tick identification were considered. More than 60 species of the family Ixodidae are present in Europe. The morphological identification of ticks is not trivial, as some species form complexes with cryptic or sibling species, such as the Rhipicephalus sanguineus group (Dantas-Torres et al., 2013) or show a large range of morphological variability, which is not captured by untrained researchers, for example the genus Hyalomma. Over this background of unstable criteria for tick identification, new species are being recognised or interspecific hybridization between taxa is reported, a biological event that may pose additinal difficulties for specific identification, even when using molecular markers (Kovalev et al., 2015). Moreover, morphological keys for ticks commonly cover only the species of medical interest, and don't always include all the stages (Arthur, 1963; Nosek and Sixl, 1972; Cordas et al., 1993; Hillyard, 1996; Filippova, 1997; Manilla, 1998; Estrada-Peña et al., 2004; Cringoli et al., 2005; Pérez-Eid, 2007). Additionally, some of them may be unreliable because they do not include the most recent concepts about the species identity. Therefore, recent studies on comparative morphology of ticks in Europe are scarce (Heylen et al., 2014).

The availability of different methods has provided insights in the use of cuticular hydrocarbon composition (Estrada-Peña et al., 1996) or MALDI-TOF (matrix assisted laser desorption ionizationtime of flight mass spectrometry) (Yssouf et al., 2013) for the identification of ticks, while most reports focused on the use of adequate genetic markers, like 16S rRNA, 12S rRNA, or cytochrome c oxidase I (coxI). Although these technologies are useful, they will always rely on reference specimens for which morphological identification needs to be correctly conducted (Nava et al., 2009; Araya-Anchetta et al., 2015). “Garbage sequences" obtained from unreliably identified specimens that accumulate in databanks are a source of molecular misidentification. They may introduce a background noise when included in the context of a phylogenetic reconstruction tick species (Zhang and Zhang, 2014) or produce an incorrect identification of individual specimens.

Several points justify the assessment of the comparative capacity of researchers working on the identification of ticks, namely: i) the specific associations between certain tick species and medically significant pathogens; ii) the concern for the spread of ticks beyond their historical ranges; and iii) the importance of observing harmonised criteria for the identification of ticks. Blind tests of quality assessment are often applied for the unbiased determination of events in different facets of the science. The protocol involves the distribution of specimens to participating laboratories by a validating team, who establish the required standard and collate and circulate the results (Ellis and Cross, 1981). Biological sciences have benefited from blind tests for the identification of organisms, the procedure being generally applied to compare the degree of similarity between the opinions of several specialists about the classification of organisms. Most tests of nonmorphological methods used for the identification of some parasitic arthropods always used the blind approach, comparing the results of a candidate method against the background of morphological identification by specialists (i.e. Dieme et al., 2014; Yssouf et al., 2013). Examples of this approach to ticks so far cover the use of DNA-barcoding for the detection of the blood meal source (Gariepy et al., 2012). Nevertheless, the morphological identification of ticks is routinely used in laboratories around the world, more commonly than molecular methods, mainly in large sample sets, which simplify the flow of work and reduces the costs. In summary, there is no objective measure of the comparative reliability of researchers in the field in recognising common species of ticks in a large region.

We aimed to candidly test the comparative performance of 14 teams of researchers involved in the study of ticks and tickborne pathogens in Europe on the morphological identification of 11 species of ticks reported as established in Europe and Northern Africa. Our study aims to identify the challenges in Europe when dealing with the identification of ticks, the causes for misidentifications, and the best procedures for harmonised results. The selection of particular species of ticks was initially done by morphological identification by three co-authors possessing marked taxonomic expertise, followed by confirmation using molecular methods by a fourth co-author (the validating team) and then distribution for performance assessment to 14 laboratories with relevant research interests in ticks. Special attention was paid to reliability of the identification according to the distribution of the ticks (e.g. species existing in the country of residence of the participant, and therefore familiar to the researcher) and the approach used in the identification of the specimens (e.g. using dedicated books, reprints, voucher specimens, etc.). A secondary aim was to estimate the confidence of the participants with their identifications as compared with the rate of mismatches, a ratio that expresses how accurately they can identify species not encountered before. We further evaluated the reliability and usability of a comprehensive key for all the species of ticks reported in the Western Palearctic as a means of increasing accuracy of identification. 
Table 1

PCR primer pairs and conditions used for the genetic identification of ticks.

\begin{tabular}{|c|c|c|c|c|}
\hline Taget gene & Primer sequence $\left(5^{\prime}->3^{\prime}\right)$ & $\begin{array}{l}\text { Melting temperature } \\
\left({ }^{\circ} \mathrm{C}\right)\end{array}$ & Fragment size (base pairs) & Reference \\
\hline $16 S$ rRNA & $\begin{array}{l}\text { 16S + 1: } \\
\text { CTGCTCAATGATTTTTTAAATTGCTGTGG } \\
\text { 16S - 1: CCGGTCTGAACTCAGATCAAGT }\end{array}$ & $\begin{array}{l}48 \\
54\end{array}$ & 456 & Black and Piesman (1994) \\
\hline $12 S$ rRNA & $\begin{array}{l}\text { T1B: AAACTAGGATTAGATACCCT } \\
\text { T2A: AATGAGAGCGACGGGCGATGT }\end{array}$ & $\begin{array}{l}51 \\
53\end{array}$ & approx. 360 & Beati and Keirans (2001) \\
\hline ITS2 & $\begin{array}{l}\text { RIB-4F: } \\
\text { CCATCGATGTGAAYTGCAGGACA } \\
\text { RIB-R: } \\
\text { GTGAATTCTATGCTTAAATTCAGGGGGT }\end{array}$ & 55 & $\begin{array}{l}\text { variable, } \\
800-1100\end{array}$ & $\begin{array}{l}\text { Zahler et al. (1995); Mclain et al. } \\
\text { (1995) (from Labruna et al., 2002) }\end{array}$ \\
\hline
\end{tabular}

\section{Material and methods}

\subsection{Species of ticks}

For an adequate representation of the most common species reported in the Western Palearctic (including Northern Africa), the following species have been included in the test: Dermacentor marginatus (Panzer) $\left(14 \%, 13 \sigma^{\top}\right)$, Dermacentor reticulatus

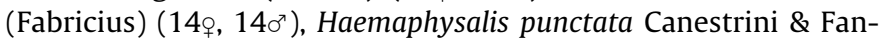
zago $\left(14 \circ, 12 \sigma^{7}\right)$, Hyalomma lusitanicum Koch (7o, $\left.11 \sigma^{7}\right)$, Hyalomma marginatum Koch $\left(14 \%, 12 \sigma^{7}\right)$, Ixodes hexagonus Leach $\left(14 \%, 13 \sigma^{7}\right.$, and 15 nymphs), Ixodes ricinus (Linnaeus) (14ㅇ, 11 $\sigma^{7}$, and 12 nymphs), Rhipicephalus annulatus (Say) (14우, 11 $\left.\sigma^{7}\right)$, Rhipicephalus bursa Canestrini \& Fanzago $\left(12 \circ, 12 \sigma^{7}\right)$, Rhipicephalus rossicus Yakimov and Kohl-Yakimova (12o, 15 $\left.\sigma^{\top}\right)$, and Rhipicephalus sanguineus s.l. $\left(9 \circ, 17 \sigma^{7}\right)$. The test was explicitly focused on the tick fauna from the two large biogeographical regions of the target territory: the countries bordering the Mediterranean basin, which also include species from Northern Africa, and countries in Central and Northern Europe. We did not include tick species that are restricted to a limited region (i.e. Ha. hispanica Gil-Collado, R. pusillus Gil-Collado, I. ventalloi Gil-Collado, I. lividus Koch) or species inadequately described or rarely reported (i.e. I. eldaricus Dzhaparidze, I. festai Tonelli-Rondelli, I. kaiseri Arthur). The species chosen for the study have special significance in both human and animal health, and they are well-known vectors of pathogens to humans or animals (Jongejan and Uilenberg, 2004).

The standardization of the batches of ticks circulated to the participants was a point of special concern. This prevented the inclusion of some species of potential importance in the test, for example Ixodes persulcatus Schulze, because the available specimens were in a variable degree of repletion, or obtained from a wide variety of sources, far from the standards required for the protocol. Only unengorged ticks were used. Specimens were always collected while questing to avoid the distortion of morphological proportions. In the case of $R$. annulatus, which is a one host species, specimens were collected as engorged nymphs on cattle, and allowed to moult in the laboratory to flat adults. Although this is not always a routine procedure during sample tick identification, we have considered this approach to provide participants with a more homogeneous sample batch, in which all the tick specimens are unengorged. All the specimens of the same species were collected in the same locality and in the same sampling event, to obtain the most homogeneous sample set possible. Every specimen with morphological abnormalities was removed from the test. Specimens of $R$. sanguineus s.l. were collected on the walls of a kennel in the Mediterranean coast of Spain, to ensure only specimens conforming to the classic description by Filippova (1997). This description overlaps with that for the morphology of the "type II" specimens reported by Dantas-Torres et al. (2013).

\subsection{Initial identification and further validation of the ticks}

All specimens $(n=306)$ were determined by one of three coauthors (none of these participated in the blind test) and confirmed by the two others. A fourth co-author enrolled in the blind test, performed a molecular determination of every species, and results were $100 \%$ in agreement with the morphological determination of the three co-authors mentioned above. The molecular identification was done by a PCR targeting the tick mitochondrial 16S ribosomal RNA gene (16S rRNA) (Black and Piesman, 1994). For some specimens, e.g. tick species in which this gene fragment had not been characterized, or was not specific enough, PCR assays for the mitochondrial 12S rRNA gene and the nuclear 5.8S-28S rRNA intergenic transcribed spacer 2 (ITS2) were also performed (Beati and Keirans, 2001; Labruna et al., 2002). In the case of $R$. sanguineus s.l. the molecular identity of the specimens confirmed that they belonged to the so-called temperate clade. The genes targeted and the primers used for molecular identification are included in Table 1 . Table 2 includes the maximum identities of sequences obtained in this study for the molecular identification of the ticks.

\subsection{Sample randomization}

The distribution of samples to each participant was blind and random. Once each individual tick had been identified by the validating team, it was placed separately in a small vial containing $70 \%$ ethanol. A unique code was randomly allocated to each specimen (vial). The specimens were sent to one of the participating laboratories which were identified by a random number. Each participant received 22-25 specimens. Because of the random nature of the test, a participant could receive several specimens of the same species, without regard to tick gender or stage. Participants were informed of this characteristic of the test, and instructed that even if a species had already been identified, the same species could appear again, or not, in the received material.

Sixteen researchers, from 14 laboratories (one of them involved only in the molecular identification) enrolled for the test. All of them have a longstanding experience in tick research, either because they work primarily on the ecology of ticks, in determination of tick-transmitted pathogens, or are involved in issues of animal and/or public health, and have a background of peerreviewed publications on the topic. Only three of the authors (those that conducted the primary/initial identification of ticks) were aware of the identity of the other participants, to avoid exchange of information during the blind test. Only one of the authors knew the complete correspondence between species of ticks, identification numbers of vials, and details of the enrolled laboratories.

\subsection{Identification of ticks}

The identification performance test comprised two steps. In the first step, identification of ticks was performed using already 
Table 2

Maximum identities of sequences obtained for the genetic characterization of tick species.

\begin{tabular}{|c|c|c|c|}
\hline \multirow[t]{2}{*}{ Tick species } & \multicolumn{3}{|c|}{ Gene sequences; \% identity \& GenBank no (no of sequences) } \\
\hline & $16 S r R N A$ & $12 S$ rRNA & ITS2 \\
\hline \multirow{2}{*}{ Dermacentor marginatus } & $99.5 \mathrm{JX} 051098(1)$ & - & - \\
\hline & $99.5 \mathrm{JX} 051097$ (1) & 100 AF031848 (1) & 99.6 FN296278 (1) \\
\hline Dermacentor reticulatus & 99.8 JF928493 (1) & - & - \\
\hline Haemaphysalis punctata & 99.5 KR870978 (3) & $100 \mathrm{AF} 483218^{\mathrm{a}}(3)$ & - \\
\hline Hyalomma lusitanicum & $99.7-100$ Z97881 (3) & - & - \\
\hline Hyalomma marginatum & $99.5 \mathrm{~L} 34307(1)$ & - & - \\
\hline Ixodes hexagonus & 100 JF928502 (1) & 99.6 AF081828 (1) & 100 GQ924083 (1) \\
\hline \multirow{4}{*}{ Ixodes ricinus } & 100 GU074616 (1) & 100 AF150029 (1) & - \\
\hline & 100 GU074606 (1) & 100 KF197118 (1) & - \\
\hline & 99.8 GU074590 (1) & 100 JN248424 (1) & - \\
\hline & 100 GU074589 (1) & - & - \\
\hline Rhipicephalus annulatus & 99.8 L34311 (2) & - & - \\
\hline Rhipicephalus bursa & 100 KU664351 (4) & 100 KC243833 (2) & - \\
\hline Rhipicephalus rossicus & 100 KP866202 $; 99.6$ KU848178 (1) & 100 AF150021 (1) & $99.3 \mathrm{AF} 271282^{\mathrm{C}}(1)$ \\
\hline Rhipicephalus sanguineus s.l. & 100 KT382469 (2) & - & - \\
\hline
\end{tabular}

a Identified as Haemaphysalis sp.

b $R$. sanguineus sequence, only a short sequence from $R$. rossicus available in GenBank (KU848178).

c Sequence identified as Rhipicephalus pumilio. No ITS2 sequence from $R$. rossicus available in GenBank.

published references, according to the decision of each participant. After the first step, the participants were requested to perform a second round of identification on the same set of ticks, using only the keys specifically prepared for the study, and to submit the results again. These keys were tailored for every stage and species of Ixodidae found in the Palearctic region, without illustrations. The keys (prepared by one of the co-authors) included the adults of 2 species of genus Dermacentor, 8 species of Haemaphysalis, 10 of Hyalomma, 23 of Ixodes, and 6 of Rhipicephalus, as well as keys for nymphs of 23 species of Ixodes.

We received responses from the 14 laboratories in the first round but only 11 responses in the second round. This resulted in 306 ticks identified by morphological methods in the first round and 259 in the second.

\subsection{Questionnaire}

The samples were circulated together with a printed questionnaire to be filled-in individually for each identified specimen, in both steps. These questionnaires were pre-labelled with the number of the vial, and included questions about the identity of the specimen, its gender and stage. We specifically aimed to collect details about the process of identification, involving the procedures followed by the participant regarding the use of keys/reprints/monographs, and how confident they felt about the identification. The complete questionnaire is included in the supplementary material.

\subsection{Calculation of rates of incorrect identifications and derived statistics}

We calculated total rates of incorrect identifications (misidentification rate $=$ MR) by tick species, in both rounds. Additionally, we calculated the specific MR for each genus (to evaluate whether some genera had poorer identification rates than others) and by sex (to check whether males or females had different MR). We further compared whether MR are higher for species that do not exist in the geographical area of each participating laboratory, defining as "endemic" the ticks that were reported from the country of the participant, and "non-endemic" the ticks that do not have permanent populations in that territory. In other words, we tested whether participating laboratories are able to identify potentially invasive ticks. The confidence of the participants with their identifications was compared with the rate of individual MR. This ratio expresses the satisfaction of participants even with inaccurate identifications.

Every participant was confidentially informed of his/her identification success rate, in both rounds. We did not consider that some species could be more difficult to identify than others, and therefore the misclassification rate (MR) is a crude, unweighted percentage. The relative performance of the participants is not included in this study.

\section{Results}

\subsection{Misidentification rate (MR)}

The identities of all ticks classified on morphological grounds before distribution to the participants were confirmed by molecular methods (Table 2). Regarding identification by participants made on morphological grounds, every specimen (except one) was correctly identified to genus level. Misidentifications were found only at the level of species or stage. The total specific MR in the first round was of $29.6 \%$, which decreased to $28.5 \%$ in the second round. The MR of stages was $1.6 \%$ ( 5 out of 306 ) and $0.8 \%$ ( 2 out of 259 ) in the first and second rounds, respectively. Two males of $D$. marginatus were initially considered as females during the first round of identification.

Fig. 1 shows the specific MR aggregated by genera, in both the first and second rounds. It must be noted that the number of responses by participants was lower in the second round than in the first (306 vs. 259 ticks, respectively). In the first round, the species in the genera Dermacentor and Ixodes obtained the lowest MR: 7.27\% and $13.92 \%$, respectively. However, every specimen of Dermacentor was correctly identified in the second round but the MR for genus Ixodes increased to $18.03 \%$. For these two genera, at species level, the MR varied between $5.4 \%$ for I. ricinus and $7.4 \%$ for D. marginatus or $D$. reticulatus.

Species of the genus Haemaphysalis had MR of $19.23 \%$ and $33.33 \%$ in the first and second rounds, respectively. The species of Hyalomma and Rhipicephalus had the highest MR in both rounds, with similar figures, around 36\% in Hyalomma and 54\% in Rhipicephalus. The MR by species are included in Fig. 2 . The species was adequately identified if the specimen was a male in $71 \%$ and $72 \%$ of cases (first and second round, respectively), or in $68 \%$ of the cases in females (both rounds). The MR was $100 \%$ for $R$. rossicus in the first 


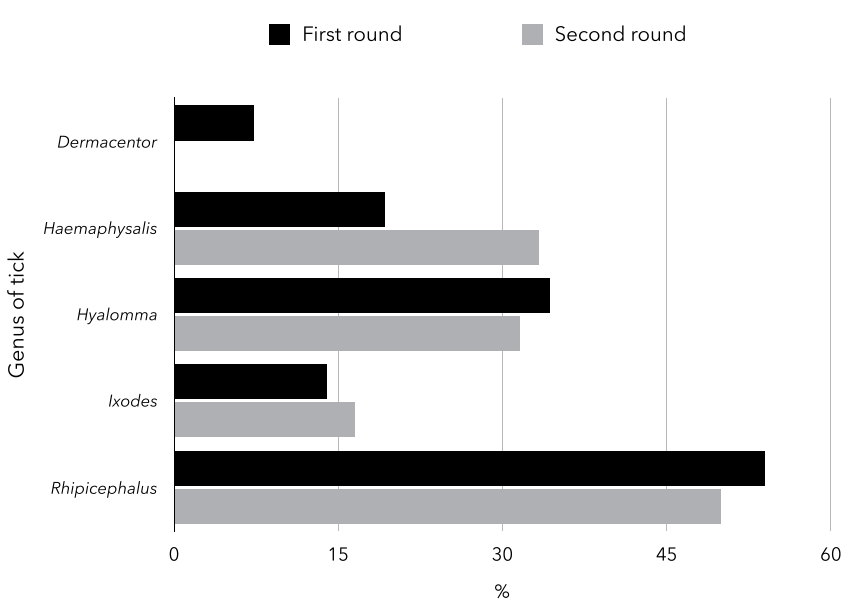

Fig. 1. The misidentification rates (MR) of ticks, by genera (in\%) in both the first and second round of morphological identification.

round, a neglected species which is rarely considered in studies in Europe.

\subsection{Correlation of the misidentification rates (MR) with the questionnaire responses}

We compared the MR with the presence/absence of the tick in the national territory of each participating laboratory. Only $8.49 \%$ of "endemic" ticks were misidentified in the first round, a value that increased to $10.45 \%$ in the second round of identifications. However, the MR of "non-endemic" ticks were $21.1 \%$ and $13.6 \%$, in the first and second rounds, respectively.

Regarding the use of bibliographical resources, $21 \%$ of specimens were identified without the help of further references, because participants were familiar with the tick, $49 \%$ used reprints for the identification (listed separately in the supplementary material), $5 \%$ used generalist book(s) that compile(s) data on species from particular regions, and 24\% used both reprints and books.

The self-perception of the participants about the reliability of identifications was variable. The participants judged that $27 \%$ of specimens had been reliably identified after a first look because they were familiar with the tick, and that $49 \%$ of specimens had been identified correctly after checking the bibliographical references. The participants had "serious concerns about the reliability

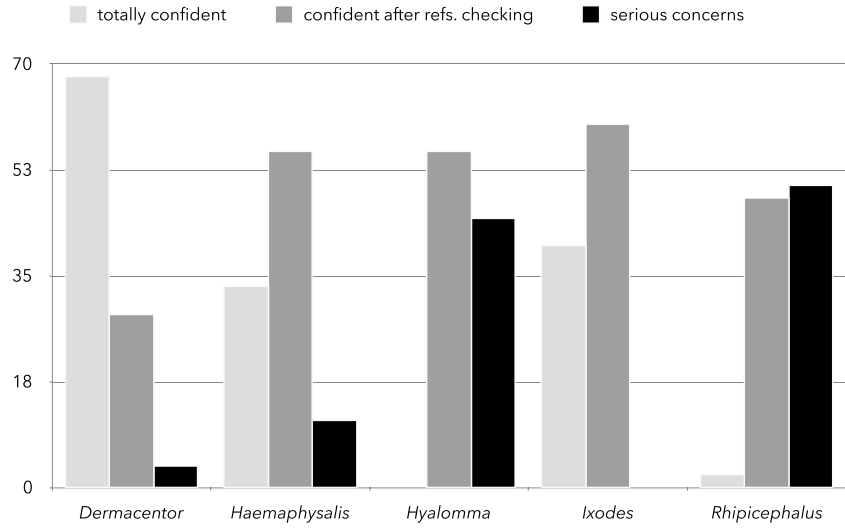

Fig. 3. The degree of self-perception by participants about the reliability of the identification of ticks, by genus of the tick (in\%).

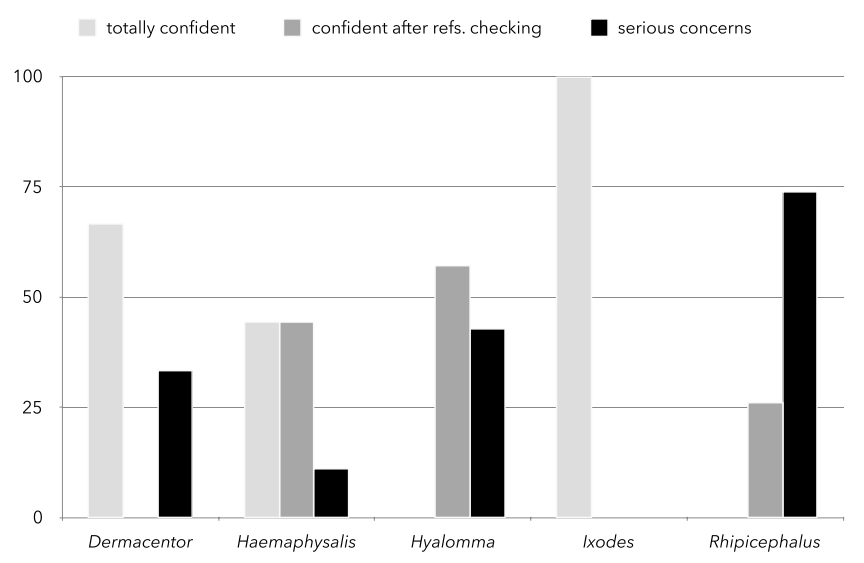

Fig. 4. The percent of erroneously identified ticks (grouped by genera) according to the self-perception by participants about the reliability of the identification of ticks.

of the morphological identification" of $23 \%$ of the specimens (see Fig. 2). Comparing these figures about self-perception with the MR, $2 \%$ of specimens were erroneously identified in the first category, $7 \%$ in the second, and $15 \%$ in the third. However, these rough figures were highly variable when considered by tick genus (Figs. 3 and 4). Most participants felt that identifications of species in genera Dermacentor and Ixodes were reliable. Self-perception

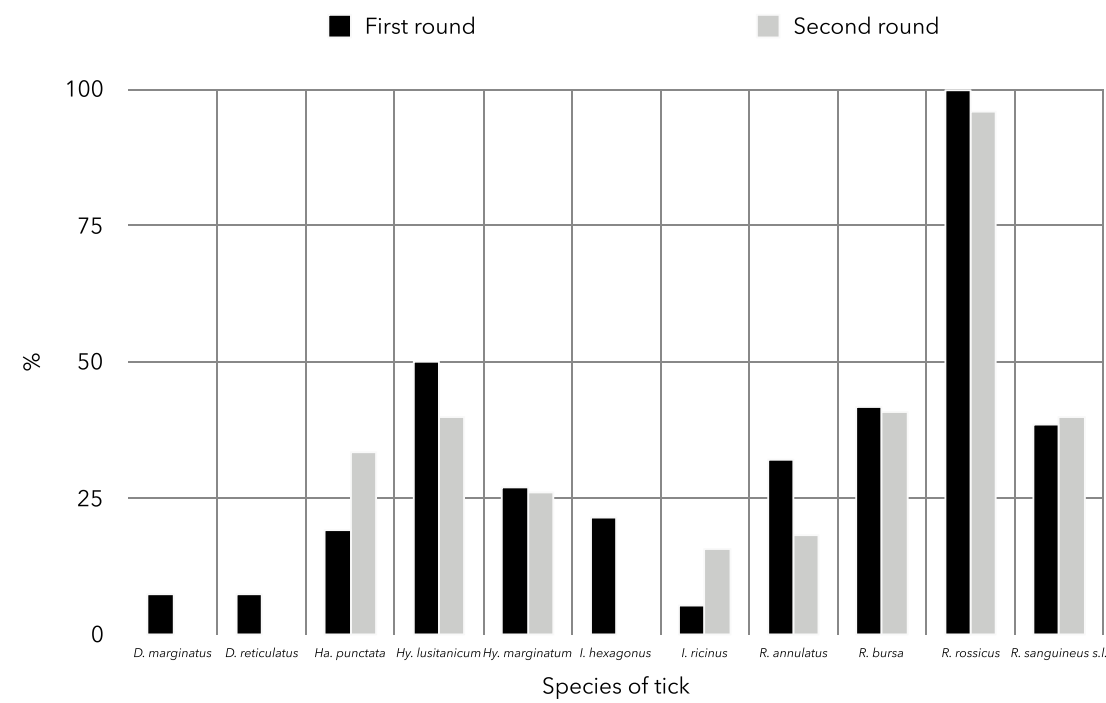

Fig. 2. The misidentification rates (MR) of species of ticks (in\%) in both the first and second round. 
of identification reliability decreased for Haemaphysalis spp. Most participants expressed "serious concerns" about the reliability of identification to species for both Hyalomma and Rhipicephalus. The comparison of these crude figures with the accuracy of the identifications provides some significant findings: participants were aware that a high percentage of the ticks in the genera Rhipicephalus and Hyalomma were probably wrongly determined.

\section{Discussion}

This study reports the results of a comparative blind test of identification of ticks carried out by 14 self-enrolled laboratories in Europe. The study was intended to evaluate the capacity to identify both the well-established species of ticks that are common in the country of the participants, and to quantify the competences of the European research teams to cope with potentially invasive species. The test included species of the genera Dermacentor, Haemaphysalis, Hyalomma, Ixodes and Rhipicephalus, distributed in Europe and Northern Africa. The study was intended to focus on the most prominent species of ixodid ticks of the target region to clearly delineate the potential of the researchers in the management of tick species with the highest impact on human or animal health.

Results showed that species in the genera Ixodes and Dermacentor had the lowest MR, while species of Hyalomma and Rhipicephalus had the highest rates of unreliable identifications. Crude results showed about $30 \%$ of misidentifications, which decreased only to $28 \%$ when a specifically-prepared key without illustrations was circulated among participants for a second round. Claims about the possibility of spread of ticks currently reported in the Mediterranean to northern latitudes (Jaenson et al., 2012) are thus a concern after these results, since $21 \%$ of these "non-endemic" species were unreliably identified.

There are several factors that could theoretically bias results in such a study as this. The most obvious is the "gold standard" established for the identity of each specimen. This was done by three coauthors, who used morphological methods. They initiated the study, distributed the specimens and did not contribute data to the identification analysis. They were aware of the geographical origin of the ticks, which other participants were not, and their identifications were confirmed $100 \%$ by a fourth participant, using molecular methods. For every other step, we adhered to the randomness of both batches of ticks and participants, therefore eliminating potential biases. Even at the stage of manuscript editing and final agreement of the submitted paper, the co-authors were unaware of the rates obtained by the other participants.

It is interesting to notice that the genera Dermacentor, Ixodes and Haemaphysalis were well-known by almost every participant, with low rates of misidentifications. However, the genera Rhipicephalus and Hyalomma accumulated the highest rates of mistakes. In other words, the purely Mediterranean species were inadequately identified by most of the participating laboratories. It is however important to mention than only 6 laboratories in Mediterranean countries were involved in the test, of a total of 14 . In the case of Rhipicephalus, the errors in classification were mainly due to the lack of a harmonised definition of the R. sanguineus s.l. group, which needs a re-description and the designation of a neotype of $R$. sanguineus S.s. (Nava et al., 2015). As a further proof of the inherent identification difficulties within the genus Rhipicephalus, no participant was able to correctly identify the adults of the neglected $R$. rossicus (rarely included in most books used by European tick researchers) in the first round, and only one laboratory managed a correct identification after a key containing the species was circulated among the participants. Therefore, we should consider that the high MR of both Hyalomma and Rhipicephalus were derived from: i) a lack of familiarity of more than $50 \%$ of participants with these ticks, ii) the deficient coverage of these species in papers commonly used for identification of ticks, iii) the unavailability of coherent criteria for identification of the species colonizing the target territory.

Of particular interest is the fact that the circulation of the keys without illustrations did not significantly improve the rate of reliable identifications, and, in some cases, introduced even higher rates of misidentifications. The second round produced poorer identification rates by some participants who had already accurately identified a specimen in the first round. The obvious interpretation is that: i) the inclusion of more species of ticks in the key produced a background noise that confused the participants (i.e. Ixodes or Haemaphysalis), ii) the lack of illustrations is a serious issue when only the crude text is used for identification.

Being unfamiliar with these "new" species, researchers tended to identify ticks by close similarity rather than by complete identity, in the absence of illustrations guiding the process. This explanation is further confirmed by the MR of "endemic" versus "non-endemic" species. The keys could probably help the participants to identify the ticks with which they were not familiar, but introduced a further complication when dealing with species which they already knew, because several such species were included in the keys adding a "background noise" in the identification.

It is necessary to stress that participants were aware in most of the cases of their unreliable identifications. Highest rates of confidence were obtained for the genera Dermacentor and Ixodes, meaning that participants were satisfied with their identifications. Highest rates of concern about the validity of identification were obtained for species of Hyalomma and Rhipicephalus. Again, there is a high agreement between the rates of concern about the validity of classifications and the actual unreliable identifications for species of these genera.

Results from this comparative test show the importance of an adequate source of information for the researchers involved in the identification of ticks. Studies in multiple fields related to ticks would benefit from adequate identifications of ticks, which should be ideally based on i) a curated library of specimens for reference, including every species and stages present at continental scale, and ii) a set of trustworthy molecular sequences either produced in house or obtained from GenBank. The $5^{\prime}$ region of the mitochondrial gene cytochrome c oxidase subunit I (coxI) is the standard marker for DNA barcoding (Hebert et al., 2004). Nevertheless, the 16S rRNA gene is a reliable marker for the tick identification at the species level (Lv et al., 2014a,b), and sequences of fragment of this gene are the most common in GenBank. Moreover, other markers such as the $12 \mathrm{~S}$ rRNA gene or internal transcribed spacers (ITS) can be complementary for tick classification (Lv et al., 2014a,b). Undoubtedly, the success of DNA barcoding for any parasite species identification relies heavily on accurate morphological identification of reference specimens. Indeed, barcoding of ticks using the molecular approach alone could lead to inconsistent results (Lv et al., 2014a) and a combination of three DNA markers (coxI, 16S rRNA, and 18S rRNA) could efficiently separate several species of ticks (Lv et al., 2014a,b). The specific identification of ticks by molecular methods should not be considered as definitive since it requires personal experience and adequate libraries, leading to the need of deposition of voucher specimens (i.e. Beati et al., 2013; Nava et al., 2014). The procedures used in the present study show that an adequate combination of several genes and of the portions that produce the highest phylogenetic information is suitable for identification of ticks.

While awareness of ticks and tick-borne pathogens increases worldwide, there is a lack of adequate knowledge about the identity of the most prominent species colonizing extensive regions in the Western Palearctic. As far as we know, a similar comparative test has never been performed in other parts of the world. It thus 
remains of interest how researchers of other regions address the issue, and how lesser known species are identified by specialists. Although other species of ticks may lack medical interest, they must be considered potentially confusing entities when compared with the focal species, introducing 'noise' in reporting. We wanted to candidly present these results, while urging the need for adequate training of experts involved in the identification of ticks, a step necessary to both address epidemiological studies and to cope with the risk posed by invasive species.

\section{Acknowledgments}

This study has been carried out under the umbrella of the European COST Action TD1303, "EurNegVec".

\section{Appendix A. Supplementary data}

Supplementary data associated with this article can be found, in the online version, at http://dx.doi.org/10.1016/j.ttbdis.2017.03. 001.

\section{References}

Araya-Anchetta, A., Busch, J.D., Scoles, G.A., Wagner, D.M., 2015. Thirty years of tick population genetics: a comprehensive review. Inf. Gen. Evol. 29, 164-179.

Arthur, D.R., 1963. British Ticks. Butterworth \& Co (Publishers) Ltd., London (218 pp).

Beati, L., Keirans, J.E., 2001. Analysis of the systematic relationships among ticks of the genera Rhipicephalus and Boophilus (Acari: ixodidae) based on mitochondrial 12S ribosomal DNA gene sequences and morphological characters. J. Parasitol. 87, 32-48.

Beati, L., Nava, S., Burkman, E.J., Barros-Battesti, D.M., Labruna, M.B., Guglielmone A.A., Faccini, J.L., 2013. Amblyomma cajennense (Fabricius, 1787) (Acari: ixodidae), the Cayenne tick: phylogeography and evidence for allopatric speciation. BMC Evol. Biol. 13 (1).

Black, W.C., Piesman, J., 1994. Phylogeny of hard- and soft-tick taxa (Acari: ixodida) based on mitochondrial 16S rDNA sequences. Proc. Nat. Acad. Sci. U. S. A. 91, $10034-10038$

Cordas, T., Aeschlimann, A., Morel, P.C., 1993. Étude morphologique des ixodidae s. str. (Schulze: 1937) de Suisse au microscope électronique à balayage. Acarologia 34, 21-46.

Cringoli, G., Lori, A., Rinaldi, L., Veneziano, V., Genchi, C., 2005. Mappe Parassitologiche - Zecche. Series Edit. Napoli, Giuseppe Cringoli.

Dantas-Torres, F., Latrofa, M.S., Annoscia, G., Giannelli, A., Parisi, A., Otranto, D., 2013. Morphological and genetic diversity of Rhipicephalus sanguineus sensu lato from the New and Old Worlds. Parasit Vectors 6, 213

Dieme, C., Yssouf, A., Vega-Rúa, A., Berenger, J.M., Failloux, A.B., Raoult, D., Almeras, L., 2014. Accurate identification of Culicidae at aquatic developmental stages by MALDI-TOF MS profiling. Parasit Vectors 7, 544

Ellis, D.V., Cross, S.F., 1981. A protocol for inter-laboratory calibrations of biological species identifications (Ring Tests). Water Res. 15, 1107-1108.

Estrada-Peña, A., de la Fuente, J., 2014. The ecology of ticks and epidemiology of tick-borne viral diseases. Antiviral Res. 108, 104-128.

Estrada-Peña, A., Gray, J.S., Kahl, O., 1996. Variability in cuticular hydrocarbons and phenotypic discrimination of Ixodes ricinus populations (Acarina: ixodidae) from Europe. Exp. Appl. Acarol. 20, 457-466.

Estrada-Peña, A., Bouattour, A., Camicas, J.-L., Walker, A.R., 2004. Ticks of Domestic Animals in the Mediterranean Region. University of Zaragoza.
Filippova, N.A., 1997. Fauna of Russia and Neighbouring Countries. Arachnoidea, Ixodid Ticks of Subfamily Amblyomminae, Vol IV (5). Russian Academy of Sciences, Zoological Institute, New Series 145, Nauka Publishing House, St Petersburg.

Gariepy, T.D., Lindsay, R., Ogden, N., Gregory, T.R., 2012. Identifying the last supper: utility of the DNA barcode library for bloodmeal identification in ticks. Mol. Ecol. Res. 12, 646-652.

Hebert, P.D., Stoeckle, M.Y., Zemlak, T.S., Francis, C.M., 2004. Identification of birds through DNA barcodes. PLoS Biol. 2, e312.

Heylen, D., De Coninck, E., Jansen, F., Madder, M., 2014. Differential diagnosis of three common Ixodes spp. ticks infesting songbirds of Western Europe: ixodes arboricola, I. frontalis and I. ricinus. Ticks Tick-borne Dis. 5, 693-700.

Hillyard, P.D., 1996. Ticks of North-West Europe. Field Studies Council, UK.

Jaenson, T.G., Jaenson, D.G., Eisen, L., Petersson, E., Lindgren, E., 2012. Changes in the geographical distribution and abundance of the tick Ixodes ricinus during the past 30 years in Sweden. Parasit Vectors 5, 1.

Jongejan, F., Uilenberg, G., 2004. The global importance of ticks. Parasitology 129 (S1), S3-S14.

Kovalev, S.Y., Mikhaylishcheva, M.S., Mukhacheva, T.A., 2015. Natural hybridization of the ticks Ixodes persulcatus and Ixodes pavlovskyi in their sympatric populations in Western Siberia. Infection. Gen. Evol. 32, 388-395.

Labruna, M.B., Marrelli, M.T., Heinemann, J.M., Fava, A.B., Cortez, A., Soares, R.M. Sakamoto, S.M., Richtzenhain, L.J., Marinotti, O., Schumaker, T.T., 2002. Taxonomic status of Ixodes didelphidis (Acari:Ixodidae). J. Med. Entomol. 39, $135-142$.

Lv, J., Wu, S., Zhang, Y., Chen, Y., Feng, C., Yuan, X., Mei, L., 2014a. Assessment of four DNA fragments (COI, 16S rDNA, ITS2, 12S rDNA) for species identification of the Ixodida (Acari: ixodida). Parasit Vectors 7, 93.

Lv, J., Wu, S., Zhang, Y., Zhang, T., Feng, C., Jia, G., Lin, X., 2014b. Development of a DNA barcoding system for the Ixodida (Acari: ixodida). Mitochondrial DNA 25, 142-149.

Maioli, M., Galletti, G., Calzolari, M., Biasi, G., Galletti, E., Dottori, M., 2012. Systematic Literature Review on the Occurrence of Ticks and Tick-borne Pathogens in the EU and Mediterranean Basin. EFSA Supporting Publications, pp. 9.

Manilla, G., 1998. Fauna d'Italia, vol. 36. Edizioni Calderini.

Mclain, D.K., Wesson, D.M., Oliver, J.H., Collins, F.H., 1995. Variation in ribosomal DNA internal transcribed spacers 1 among eastern populations of Ixodes scapularis (Acari: ixodidae). J. Med. Entomol. 32, 353-360.

Nava, S., Guglielmone, A.A., Mangold, A.J., 2009. An overview of systematics and evolution of ticks. Frontiers Biosci. 14, 2857-2877.

Nava, S., Beati, L., Labruna, M.B., Cáceres, A.G., Mangold, A.J., Guglielmone, A.A., 2014. Reassessment of the taxonomic status of Amblyomma cajennense (L) with the description of three new species, Amblyomma tonelliae n. sp., Amblyomma interandinum n. sp. and Amblyomma patinoi n. sp., and reinstatement of Amblyomma mixtum, and Amblyomma sculptum (Ixodida: ixodidae). Ticks Tick-borne Dis. 5, 252-276.

Nava, S., Estrada-Peña, A., Petney, T., Beati, L., Labruna, M.B., Szabó, M.P., Guglielmone, A.A., 2015. The taxonomic status of Rhipicephalus sanguineus (Latreille, 1806). Vet. Parasitol. 208, 2-8.

Nosek, J., Sixl, W., 1972. Central european ticks (Ixodoidea): key for determination. Jahrbuch der naturwissenschaftlichen Abteilung Joanneum 1, 61-92.

Pérez-Eid, C., 2007. Les tiques: identification, biologie, importance médicale et vétérinaire. Lavoisier Eds. 314pp.

Yssouf, A., Flaudrops, C., Drali, R., Kernif, T., Socolovschi, C., Berenger, J.M., Parola, P., 2013. Matrix-assisted laser desorption ionization-time of flight mass spectrometry for rapid identification of tick vectors. J. Clin. Microbiol. 51, 522-528.

Zahler, M., Gothe, R., Rinder, H., 1995. Genetic evidence against a morphologically suggestive conspecificity of Dermacentor reticulatus and D. marginatus (Acari: ixodidae). Int. J. Parasitol. 25, 1413-1419.

Zhang, R.L., Zhang, B., 2014. Prospects of using DNA barcoding for species identification and evaluation of the accuracy of sequence databases for ticks (Acari: ixodida). Ticks Tick-borne Dis. 5, 352-358. 